\title{
Ferrimagnetism in the organic polymeric Hubbard model: Quantum Monte Carlo simulation
}

\author{
Shi-Dong Liang and Z. D. Wang \\ Department of Physics, University of Hong Kong, Pokfulam Road, Hong Kong, China
}

Qianghua Wang

Physics Department and National Laboratory of Solid State Microstructures, Institute for Solid State Physics, Nanjing University, Nanjing 210093, People's Republic of China,

and Center for Advanced Studies in Science and Technology of Microstructures, Nanjing 210093, People's Republic of China

Shun-Qing Shen

Department of Physics, University of Hong Kong, Pokfulam Road, Hong Kong, China

(Received 18 June 1998)

\begin{abstract}
The ground-state properties of organic polymers are studied by means of the quantum Monte Carlo simulation. The polymer doped by transition-metal impurities at every other radical site of the chain is described by the quasi-one-dimensional polymeric Hubbard chain. The topological structure of the chain possesses a flatband structure of the energy band. The spin-spin correlation function and the static magnetic susceptibility are investigated in the case of half filling. Our analysis shows that the on-site Coulomb repulsions in the chain and/or in the radical lead to the coexistence of ferromagnetic and antiferromagnetic order, i.e., the ferrimagnetic order. The on-site Coulomb repulsion $\left(U_{d}\right)$ of electrons at the radicals plays a more significant role in stabilizing the ferromagnetic order than that $(U)$ on the chain does, while $U$ has a stronger impact on the antiferromagnetic order. [S0163-1829(99)04205-8]
\end{abstract}

Since the discovery of ferromagnetism in organic polymers, such as poly-BIPO, ${ }^{1-3}$ m-PDPC, ${ }^{4}$ and p-NPNN,${ }^{5}$ there has been considerable interest in this kind of magnetism in organic molecules. ${ }^{4-8}$ The polymer chain, m-PDPC, is described by the Kondo-Hubbard Hamiltonian, which exhibits the ferrimagnetic order by means of the mean-field theory ${ }^{4}$ and the quantum Monte Carlo simulation. ${ }^{6}$ For the polyBIPO of polymers, the primary zigzag chain consists of $\pi$-conjugated carbon atoms and a radical $(R)$ at every other site shown in Fig. 1. One attributes this kind of organic ferromagnetism to the polymer chain doped by the transitionmetal impurities at the radical sites in which unpaired $d$ or $f$ electrons interact with the itinerant $\pi$ electrons along the chain. Early theoretical efforts ${ }^{7}$ are based on the $\mathrm{Su}-$ Schrieffer-Heeger model supplemented by a quasi-classical spin coupling between the $\pi$ electron and the $d$ electron at the radical. The result of Hartree-Fock approximation indicates that a stable ferromagnetic order could occur, ${ }^{7}$ and the quantum Monte Carlo simulation shows the transition between the charge-density wave and the spin-density wave (SDW) when the nearest-neighbor interaction is taken into account. ${ }^{8}$ However, it appears to be a quite crude approximation to employ only Ising-like spin coupling in Refs. 4, 7, and 8 to describe the interaction between $s$ electrons in the chain and $d$ electrons in the radical. In this paper, we suggest that this kind of polymer chain be modeled by the polymeric Hubbard Hamiltonian, without any a priori restriction on the radical spin projection. ${ }^{4,7,8}$ Although the value of the total spin of this model can be obtained exactly, ${ }^{9,10}$ some other interesting properties, such as antiferromagnetic order/SDW and the effect of the impurities at the radical, will be elucidated by using the quantum Monte Carlo method. In particular, it is numerically shown that the Coulomb repulsion ei- ther in the radical or in the chain leads to the coexistence of the ferromagnetic and antiferromagnetic order, i.e., the ferrimagnetic order.

It is well known that a one-dimensional chain is unstable against the dimerization effect at low temperatures when the electron-phonon interaction is taken into account. However, here we focus attention on the detailed magnetic structure when the polymer chain is doped by transition-metal impurities at the radical. Since unpaired $d$ or $f$ electrons at the radical interact with the itinerant $\pi$ electrons in the chain (shown in Fig. 1), this quasi-one-dimensional system can be described by the polymeric Hubbard Hamiltonian

$$
\begin{aligned}
H= & -\sum_{\langle i, j\rangle, \sigma} t_{i}\left(C_{i, \sigma}^{\dagger} C_{j, \sigma}+\text { H.c. }\right) \\
& +\sum_{i} U_{i}\left(n_{i, \uparrow}-\frac{1}{2}\right)\left(n_{i, \downarrow}-\frac{1}{2}\right)-\mu \sum_{i, \sigma} n_{i, \sigma},
\end{aligned}
$$

where $t_{i}=t$ and $t_{d}$ are the hopping terms along the chain and between the chain and the radical, respectively; $U_{i}=U$ and $U_{d}$ are the on-site Coulomb repulsions for the $\pi$ electrons on the chain and the $d$ electron at the radical. The sum $\langle i, j\rangle$ runs over all pairs of nearest-neighbor lattice sites. We set the number of the lattice sites to be $N: 2 N / 3$ of them are in the chain and $N / 3$ in the radical depicted in Fig. 1. $C_{i, \sigma}^{\dagger}\left(C_{i, \sigma}\right)$ are the creation (annihilation) operators for electrons with spin $\sigma$ at lattice site $i$, and $n_{i}=\Sigma_{\sigma} n_{i \sigma}=\Sigma_{\sigma} C_{i \sigma}^{+} C_{i \sigma} \cdot \mu$ is the chemical potential.

The topological structure of the polymer forms a bipartite lattice. The hopping terms just connect the sites belonging to different sets of sublattices. For the noninteracting case, the 


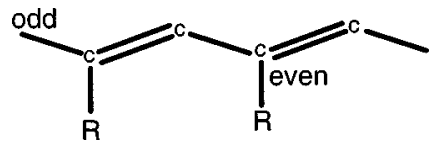

FIG. 1. The sketched structure of poly-BIPO. $R$ denotes a kind of side-free radical containing a $d$ electron.

Hamiltonian can be diagonalized by using the Fourier transformation. The single-particle energy spectrum is obtained by

$$
\boldsymbol{\epsilon}_{k}^{\sigma}=\left\{\begin{array}{l}
0, \\
\pm \sqrt{4 t^{2} \cos (k)^{2}+t_{d}^{2}}
\end{array}\right.
$$

where $k=(\pi / N / 3) n$ with $n=0, \ldots,(N / 3)-1$. The threeband structure originates from the asymmetric property of the bipartite lattice structure. Each unit cell contains three different species of sites. As each radical connects only one site on the chain and there is no hopping between these radicals, this feature leads to the appearance of a flat band. The $k$ states of this flat band look still like the Bloch waves. There are the band gap $\Delta=t_{d}$ between the flat band and other two bands. It is worth mentioning that the flat band leads to a singularity of the density of states near the Fermi energy when the system is half filled. When the electron-electron interaction $U$ is turned on, the magnetic susceptibility becomes divergent at Fermi surface, which means that the ground state is unstable and could evolve into a ferromagnetic order at half filling. For $t_{d}=0$, the band gap disappears and the system reduces to an exact one-dimensional tightbinding chain with the single-electron energy $\epsilon_{k}^{\prime}=$ $-2 t \cos \left(k^{\prime}\right)$ with $k^{\prime}=(\pi / N / 3) n^{\prime}$ with $n^{\prime}=0, \ldots,(2 N / 3)$ -1 . In general cases, $t \neq t_{d}$ and $U \neq U_{d} \neq 0$, the spin splitting can occur, which may lead to the magnetic order or SDW for half fillings. Obviously, it is extremely difficult to solve the model rigorously even though some exact results of the ground state have been obtained. ${ }^{9,10}$ Nevertheless, we can use the grand-canonical ensemble quantum Monte Carlo method $^{11,12}$ (GCMC) to study the low-temperature properties of the model.

The partition function of the system is written as

$$
\begin{aligned}
Z & =\operatorname{Tr}\left(e^{-\beta H}\right) \\
& =\operatorname{Tr}\left(e^{-\Delta \tau(K+V)}\right)^{L},
\end{aligned}
$$

where the inverse temperature $\beta$ is decomposed into $\Delta \tau L$ by introducing a small imaginary-time step $\Delta \tau$. The kinetic and potential terms are

$$
\begin{aligned}
K & =-\sum_{\langle i, j\rangle, \sigma} t_{i}\left(C_{i, \sigma}^{\dagger} C_{j, \sigma}+C_{j, \sigma}^{\dagger} C_{i, \sigma}\right)-\mu \sum_{i, \sigma} n_{i, \sigma} \\
& =\sum_{\langle i, j\rangle, \sigma} C_{i, \sigma}^{\dagger} k_{i j} C_{j, \sigma}
\end{aligned}
$$

and

$$
V=\sum_{i} U_{i}\left(n_{i, \uparrow}-\frac{1}{2}\right)\left(n_{i, \downarrow}-\frac{1}{2}\right),
$$

respectively. By using the Hubbard-Stratonovich transformation,

$$
\begin{aligned}
& e^{-\Delta \tau U_{i}\left(n_{i, \uparrow}-1 / 2\right)\left(n_{i, \downarrow}-1 / 2\right)} \\
& \quad=\frac{1}{2} e^{-\Delta \tau U_{i} / 4} \sum_{s_{i, l}= \pm 1} \exp \left[-\Delta \tau s_{i, l} \lambda_{i}\left(n_{i, \uparrow}-n_{i, \downarrow}\right)\right]
\end{aligned}
$$

at each lattice site $i$ and each imaginary-time slice $l . \lambda_{i}$ satisfies $\cosh \left(\Delta \tau \lambda_{i}\right)=\exp \left(\Delta \tau U_{i} / 2\right)$, which depends on the lattice site, i.e., $U_{i}=U$ and $U_{d}$ correspond to the sites along the chain and at the radical, respectively. The interaction terms, $\exp (-\Delta \tau V)$, can be expressed in terms of quadratic fermion operator with Ising spin field $\left(s_{i, l}\right)$, where $s_{i, l}= \pm 1$. As a result, the trace over electron degrees of freedom can be rewritten as ${ }^{11,12}$

$$
Z=\sum_{s_{i, l}= \pm 1} \operatorname{det} M^{+} \operatorname{det} M^{-}
$$

with

$$
M^{\sigma}=\left(I+B_{L}^{\sigma} B_{L-1}^{\sigma}, \ldots, B_{1}^{\sigma}\right)
$$

and

$$
B_{l}^{\sigma}=\exp [-\Delta \tau(k+\sigma v(l))]
$$

where $v(l)=\delta_{i, j} \lambda_{i} s_{i, l}$ and $I$ is the $N \times N$ unit matrix. According to the algorithm of GCMC in Refs. 12 and 13, we yield a sequence of spin configurations by updating the Ising spins. The transition probability can be expressed in terms of the equal-time Green's function, $G^{\sigma}(l)_{i, j}$ $=\left\langle T\left[C_{i, \sigma}(l \Delta \tau) C_{j, \sigma}^{+}(l \Delta \tau)\right]\right\rangle$, which is updated spatially by the Dyson's equation ${ }^{12}$ while the Ising spin is flipped. The time evolution of Green's function is based on $G^{\sigma}(l+1)$ $=B_{l+1}^{\sigma} G^{\sigma}(l) B_{l+1}^{\sigma-1}$. We use the matrix-decomposition technique $^{12}$ to reduce the instability of the Green's function at low temperatures.

In the half-filled case, the particle-hole symmetry implies that the product of determinants for the two spin species is positive semidefinite for arbitrary configurations so that there is no sign problem. The physical observables, based on this procedure, can be expressed in terms of the single-particle Green's function $\left\langle C_{i} C_{j}^{\dagger}\right\rangle$.

We have performed simulations in the half-filled case for the lattice-size up to 24 sites with the periodic boundary condition (setting $t=1$ ). The time slice was taken to be $\Delta \tau$ $=0.0625-0.25$. Simulations were run at least 5000 sweeps to obtain the measurements after thermalizing the system. In the presented figures, the lines are used as a guide for the eye, and the statistical errors are within the corresponding symbol size.

Since the value of the total spin is proved to be $S=N / 6$ in the ground state, 9,10 we can examine our Monte Carlo codes by calculating the square of total spin, which is defined by ${ }^{10}$ 

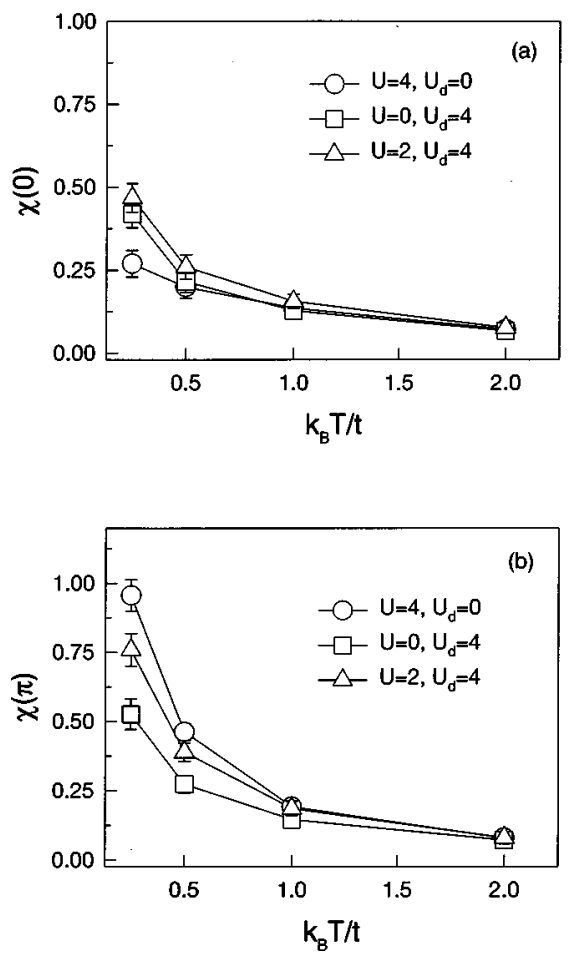

FIG. 2. The magnetic susceptibilities (a) $\chi(0)$ and (b) $\chi(\pi)$ vs temperature in the case of $t_{d}=0.2$ and $N=24$.

$$
\begin{aligned}
\left\langle\mathbf{S}^{2}\right\rangle & =\sum_{i, j}\left\langle\mathbf{S}_{\mathbf{i}} \cdot \mathbf{S}_{\mathbf{j}}\right\rangle \\
& =\sum_{i, j}\left[\frac{1}{2}\left\langle S_{i}^{+} S_{j}^{-}+S_{i}^{-} S_{j}^{+}\right\rangle+\left\langle S_{i}^{z} S_{j}^{z}\right\rangle\right],
\end{aligned}
$$

where $S_{i}^{+}=C_{i, \uparrow}^{\dagger} C_{i, \downarrow}, S_{i}^{-}=C_{i, \downarrow}^{\dagger} C_{i, \uparrow}$, and $S_{i}^{z}=\frac{1}{2}\left(n_{i, \uparrow}-n_{i, \downarrow}\right)$. For example, in the cases of $U=U_{d}=2$ for $N=6,12$, and 24 , we confirmed that the average spin per site approaches the rigorous result $1 / 6$ at sufficient low temperatures $\left(k_{B} T / t\right.$ $<0.065)$, while in the case of $U=U_{d}=0$, the total spin is close to zero at low temperature as expected.

To study the detailed magnetic order in the ground state, we calculate the zero-frequency susceptibility versus temperature (Fig. 2), which is defined as

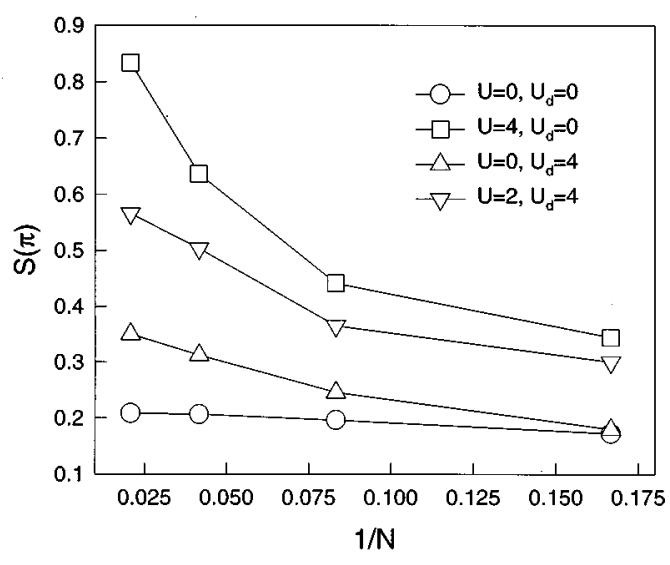

FIG. 3. The finite-size scalings on the magnetic structure factor $S(\pi)$ for $t_{d}=0.2$ at $\beta=N$.

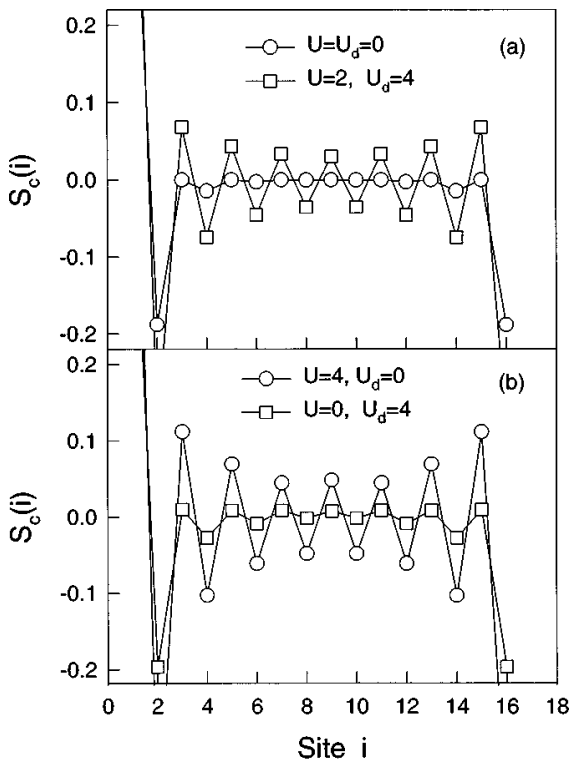

FIG. 4. The spin-spin correlation function $S_{c}(l)$ along the chain in the cases of (a) $U=U_{d}=0$ and $U=2$ with $U_{d}=4$, (b) $U=4$ with $U_{d}=0$ and $U=0$ with $U_{d}=4$ for $t_{d}=0.2$ at $\beta=N=24$.

$$
\chi(q)=\frac{1}{N^{2}} \sum_{i, j} e^{i q\left(R_{i}-R_{j}\right)} \int_{0}^{\beta} d \tau\left\langle S_{i}^{z}(\tau) S_{j}^{z}\right\rangle .
$$

For $q=0$ it satisfies

$$
\chi(q=0)=\beta S(q=0),
$$

where $S(q)$ is the magnetic structure factor,

$$
S(q)=\frac{1}{N^{2}} \sum_{i, j} e^{i q\left(R_{i}-R_{j}\right)}\left\langle S_{i}^{z} S_{j}^{z}\right\rangle
$$

since the total magnetization commutes with the Hamiltonian. ${ }^{11}$ The susceptibilities were evaluated by using Eq. (12) for $q=0$ and $q=\pi \cdot{ }^{13}$ We consider three typical cases: ${ }^{14} t_{d}=0.2, U=4$ with $U_{d}=0$ (open circles), $U=0$ with $U_{d}=4$ (open squares), and $U=2$ with $U_{d}=4$ (open triangles). The numerical results in Fig. 2 show that $\chi(\pi)$

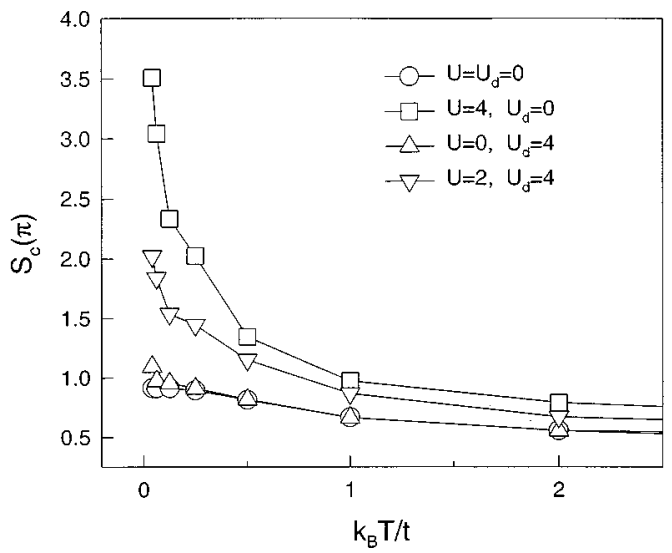

FIG. 5. The magnetic structure factor $S_{c}(\pi)$ along the chain vs temperature in the case of $t_{d}=0.2$ and $N=24$. 
$-\chi(0)>0$. It implies that the antiferromagnetic correlation is always stronger than the ferromagnetic one. The susceptibilities $\chi(0)$ and $\chi(\pi)$ increase when the temperature is lowered, and diverge ultimately when $T \rightarrow 0$. This implies that the ferromagnetic and antiferromagnetic long-range orders coexist in the ground state. Comparing the effects of $U$ and $U_{d}$ in the three cases [see Figs. 2(a) and 2(b)], we find that $U_{d}$ has more significant impact on the ferromagnetic order, while $U$ has stronger impact on the antiferromagnetic order.

In order to see whether the long-range orders survive in the thermodynamic limit, we perform a finite-size scaling analysis $(N=6,12,24,48$, and $\beta=N)$ on the magnetic structure factors $S(\pi)$ in Fig. 3. $S(\pi)$ increases obviously while increasing the chain size in the three typical cases of $U \neq 0$ or $U_{d} \neq 0$ (squares, upsidedown triangles, and triangles), which means that the antiferromagnetic order indeed occurs in the thermodynamic limit as long as the on-site Coulomb repulsion is turned on. In the absence of on-site Coulomb repulsion (open circles), the slope of $S(\pi)$ is almost zero. Thus there is no magnetic order. In short, the on-site Coulomb repulsion $U$ enhances the antiferromagnetic order, being in full agreement with the results in Fig. 2.

Figure 4 shows the equal-time spin-spin correlation function $S_{c}(l)=(1 / L) \Sigma_{i}^{L}\left\langle S_{i}^{z} S_{i+l}^{z}\right\rangle$ along the chain, where $L$ $=2 N / 3$ is the number of sites on the main chain. It is shown from Fig. 4 that the SDW arises in the presence of the on-site Coulomb repulsions along the chain and/or in the radical. When the on-site Coulomb repulsions (circles) are turned off the SDW disappears. Thus both $U$ and $U_{d}$ enhance the anti- ferromagnetic correlation. From Fig. 4(b), we find that $U$ plays a more important role in stabilizing the SDW than $U_{d}$ does.

In order to confirm the antiferromagnetic long-range correlation further, we plot the magnetic structure factor $S_{c}(q$ $=\pi$ ) along the chain versus temperature in Fig. 5. In the absence of $U$ and $U_{d}$ (circles in Fig. 5), the slope of $S_{c}(\pi)$ with respect to temperature is almost zero, which means that there is no SDW in the ground state. For $U=0$ and $U_{d}=4$ (triangles), $S_{c}(\pi)$ increases as temperature is lowered. When $U$ is turned on (squares and upsidedown-triangles), $S_{c}(\pi)$ becomes divergent with decreasing temperature. This indicates that there is indeed SDW along the chain.

In summary, the ground-state properties of organic polymeric Hubbard model are studied by means of the quantum Monte Carlo simulation. The doped chain possesses a flatband structure of the energy spectrum. ${ }^{15}$ We have found numerically that the on-site Coulomb repulsions ( $U$ and/or $U_{d}$ ) lead to the coexistence of ferromagnetic and antiferromagnetic orders, i.e., the ferrimagnetic order, in the ground state. $U_{d}$ plays a more significant role in stabilizing the ferromagnetic order than $U_{d}$ does, while $U$ has a stronger impact on the antiferromagnetic order.

We would like to thank Professor X. Sun for his helpful discussions. This work was supported by the RGC grant of Hong Kong, under Grant No. HKU 262/95P and by the CRCG research grant of the University of Hong Kong. Q.H.W. was supported by the National Science Foundation of China and by Sanzhu Co. Ltd. in Shandong.
${ }^{1}$ Y.V. Korshak, T.V. Medvedeva, A.A. Ovchinnikov, and V.N. Spector, Nature (London) 326, 370 (1987); A.A. Ovchinnikov and V.N. Spector, Synth. Met. 27, B615 (1988).

${ }^{2}$ Y. Cao, P. Wang, Z. Hu, S.Z. Li, L.Y. Zhang, and J.G. Zhao, Synth. Met. 27, B625 (1988).

${ }^{3}$ H. Iwamura et al., Mol. Cryst. Liq. Cryst. 125, 379 (1985).

${ }^{4}$ K. Nasu, Phys. Rev. B 33, 330 (1986).

${ }^{5}$ M. Takahashi et al., Phys. Rev. Lett. 67, 746 (1991).

${ }^{6}$ A.M.S. Maced, M.C. dos Santos, M.D. Coutinho-Filho, and C.A. Macedo, Phys. Rev. Lett. 74, 1851 (1995).

${ }^{7}$ Z. Fang, Z.L. Liu, and K.L. Yao, Phys. Rev. B 49, 3916 (1994); Z. Fang, Z.L. Liu, K.L. Yao, and Z.G. Li, ibid. 51, 1304 (1995); Z. Fang, K.L. Yao, and Z.L. Liu, Z. Phys. B 99, 425 (1996).

${ }^{8}$ Shi-Dong Liang, Qianghua Wang, and Z. D. Wang, Z. Phys. B 104, 27 (1997).

${ }^{9}$ E. Lieb, Phys. Rev. Lett. 62, 1201 (1989); S.Q. Shen, Int. J. Mod.
Phys. B 12, 709 (1998); S.Q. Shen et al., Phys. Rev. Lett. 72, 1280 (1994).

${ }^{10}$ S.Q. Shen, Phys. Rev. B 53, 14252 (1996); 54, 4397 (1996); 55, 14330 (1997).

${ }^{11}$ J.E. Hirsch, Phys. Rev. Lett. 51, 1900 (1983); Phys. Rev. B 28, 4059 (1983); 31, 4403 (1985).

${ }^{12}$ S.R. White, D.J. Scalapino, R.L. Sugar, E.Y. Loh, J.E. Gubernatis, and R.T. Scalettar, Phys. Rev. B 40, 506 (1989).

${ }^{13}$ To examine the reliability of our numerical approach, we also calculated the susceptibility for $q=0$ according to Eq. (13). The results obtained from the two different ways coincide with each other completely.

${ }^{14}$ For a realistic polymer chain, $t_{d}$ should not be too large and $U_{d}>U$.

${ }^{15}$ Shi-Dong Liang, G. Wang, Z. D. Wang, and S. A. Shen, Int. J. Mod. Phys. B 12, 2031 (1998). 